\title{
EST analysis in Ginkgo biloba: an assessment of conserved developmental regulators and gymnosperm specific genes
} Eric D Brenner*1, Manpreet S Katari², Dennis W Stevenson'1, Stephen A Rudd ${ }^{3}$, Andrew W Douglas ${ }^{1}$, Walter N Moss ${ }^{1}$, Richard W Twigg5, Suzan J Runko ${ }^{1}$, Giulia M Stellari ${ }^{6}$, WR McCombie ${ }^{4}$ and Gloria M Coruzzi ${ }^{2}$

\author{
Address: ${ }^{1}$ The New York Botanical Garden, 200th St. and Kazimiroff, Bronx, NY 10458-5126, USA, ${ }^{2}$ New York University, Department of Biology \\ 1009 Main Building, New York, NY 10003, USA, ${ }^{3}$ Centre for Biotechnology, Tykistökatu 6, FIN-20521 Turku, Finland, ${ }^{4}$ Genome Research Center, \\ Cold Spring Harbor Laboratory, 500 Sunnyside Blvd, Woodbury, NY 11797, USA, 5Biology Department, Duke University, Box 91000 , Durham, \\ North Carolina, 27708 and ${ }^{6}$ Department of Plant Biology, Cornell University, Ithaca NY 14850, USA \\ Email: Eric D Brenner* - ebrenner@nybg.org; Manpreet S Katari - mkatari@nyu.edu; Dennis W Stevenson - dws@nybg.org. \\ Stephen A Rudd - stephen.rudd@btk.utu.fi; Andrew W Douglas - adouglas@nybg.org; Walter N Moss - wmoss@nybg.org; \\ Richard W Twigg - richard.twigg@duke.edu; Suzan J Runko - srunko@nybg.org; Giulia M Stellari - gms37@cornell.edu; \\ WR McCombie - mccombie@cshl.org; Gloria M Coruzzi - gloria.coruzzi@nyu.edu \\ * Corresponding author
}

Published: 15 October 2005

BMC Genomics 2005, 6:143 doi:10.1 186/1471-2164-6-143

This article is available from: http://www.biomedcentral.com/I47/-2/64/6//43

(c) 2005 Brenner et al; licensee BioMed Central Ltd.

This is an Open Access article distributed under the terms of the Creative Commons Attribution License (http://creativecommons.org/licenses/by/2.0), which permits unrestricted use, distribution, and reproduction in any medium, provided the original work is properly cited.

\begin{abstract}
Background: Ginkgo biloba L. is the only surviving member of one of the oldest living seed plant groups with medicinal, spiritual and horticultural importance worldwide. As an evolutionary relic, it displays many characters found in the early, extinct seed plants and extant cycads. To establish a molecular base to understand the evolution of seeds and pollen, we created a cDNA library and EST dataset from the reproductive structures of male (microsporangiate), female (megasporangiate), and vegetative organs (leaves) of Ginkgo biloba.
\end{abstract}

Results: RNA from newly emerged male and female reproductive organs and immature leaves was used to create three distinct cDNA libraries from which 6,434 ESTs were generated. These 6,434 ESTs from Ginkgo biloba were clustered into 3,830 unigenes. A comparison of our Ginkgo unigene set against the fully annotated genomes of rice and Arabidopsis, and all available ESTs in Genbank revealed that 256 Ginkgo unigenes match only genes among the gymnosperms and non-seed plants - many with multiple matches to genes in non-angiosperm plants. Conversely, another group of unigenes in Gingko had highly significant homology to transcription factors in angiosperms involved in development, including MADS box genes as well as post-transcriptional regulators. Several of the conserved developmental genes found in Ginkgo had top BLAST homology to cycad genes. We also note here the presence of ESTs in G. biloba similar to genes that to date have only been found in gymnosperms and an additional 22 Ginkgo genes common only to genes from cycads.

Conclusion: Our analysis of an EST dataset from G. biloba revealed genes potentially unique to gymnosperms. Many of these genes showed homology to fully sequenced clones from our cycad EST dataset found in common only with gymnosperms. Other Ginkgo ESTs are similar to developmental regulators in higher plants. This work sets the stage for future studies on Ginkgo to better understand seed and pollen evolution, and to resolve the ambiguous phylogenetic relationship of $G$. biloba among the gymnosperms. 


\section{Background}

Ginkgo biloba is a widely popular tree that is native to China and has been cultivated for well over a millennium. In Asia, G. biloba is used medicinally and its seeds are also a popular cuisine item. In the West, Ginkgo leaf extracts are commonly used for a variety of folk remedies (for review see: [1]) including as a treatment for improving cognitive function [2,3]. Today's Ginkgo biloba is the sole surviving species of an ancient group (Ginkgophytes) of seed plants that may even date from the Permian (approximately 150-200 million years ago) [4]. The genus Ginkgo itself goes back to the Jurassic period - approximately 170 million years ago [5]. Although it is widely believed that the survival of G. biloba depended upon Buddhist monks, who venerated the tree cultivated in their temple grounds, molecular evidence suggests that some stands in China (Wuchuan, Guizhou) are of natural origin representing vestige populations [6]. As a living fossil, Ginkgo biloba has changed little in morphology from its extinct relatives [5]. Along with the Cycadales, Coniferales and Gnetales, the Ginkgoales is one of four orders of non-flowering seed plants (gymnosperms) that form a sister group to the angiosperms (Figure 1).

Morphological $[7,8]$ and molecular analysis have not yet succeeded in defining the precise phylogenetic hierarchy of the four gymnosperm clades [9]. Ginkgo potentially forms a sister group with the Coniferales (partly due to similar characteristics such as axillary branching and simple leaves). Another model, based on molecular sequence data, places Ginkgo with the Cycadales [10-12]. Interestingly cycads and Ginkgo both share certain plesiomorphic (ancestral) characters found in early fossil seed plants such as haustorial pollen $[13,14]$, which release motile male gametes [15] as well as a large four celled opening in the neck of the archegonia [13,16]. Despite the presence of these and other early seed-plant characteristics, surprisingly little work has been performed on Ginkgo and cycads. Some recent molecular [17] and genomic [18] research on cycads have been conducted and molecular studies of Ginkgo genes have been initiated as well [1921]. However, no genomic work on Ginkgo biloba has been completed to date.

To begin our genomic treatment of Ginkgo biloba, we focused our initial efforts on developing reproductive and vegetative tissues (Figure 2). Separating Ginkgo male and female structures at an early stage is straightforward because Ginkgo is strictly a dioecious plant (male and female organs on separate individuals). Organ emergence can generally be pinpointed to a specific time of the year in that both reproductive and vegetative tissues are regularly produced in the beginning of May at our collection site in New York. The reproductive structures, megasporangia bearing ovules (Figure 2A-C) (from female trees) and microsporangia bearing pollen (Figure 2D-F) (from male trees), emerge at the apex of short, determinate (spur) shoots. A discreet flush of leaves are also produced in male and female short-shoots (Figure 2A and 2D) $[13,16,22]$. Long shoots (not shown) exhibit indeterminate growth and yield only vegetative organs. Long-shoots are identifiable by their obvious longer internodes, whereas short shoots (Figure 2A and 2D) have telescoped internodes. Each season, short-shoots might exhibit extensive internode growth and be transformed into longshoots and vice versa. Consequently, reproductive shoots can become vegetative or vegetative shoots can become reproductive.

Until now, little is known regarding the genetic regulation of development in the oldest living seed plants. In order to uncover the genetic controls directing growth and development in Ginkgo biloba, we generated expressed sequence tags (ESTs) from cDNA libraries of very young, recently emerged organs of fertile short-shoots where a large number of regulatory genes are expected to be present. Below is an analysis of these ESTs from Ginkgo biloba. In all three tissues examined, vegetative, microsporangia (male), and megasporangia (female), we found a large number of ESTs with similarity to angiosperm developmental genes. Conversely, a certain number of Ginkgo biloba ESTs were uncovered with homology to genes only found in gymnosperms and non-seed plants, including a

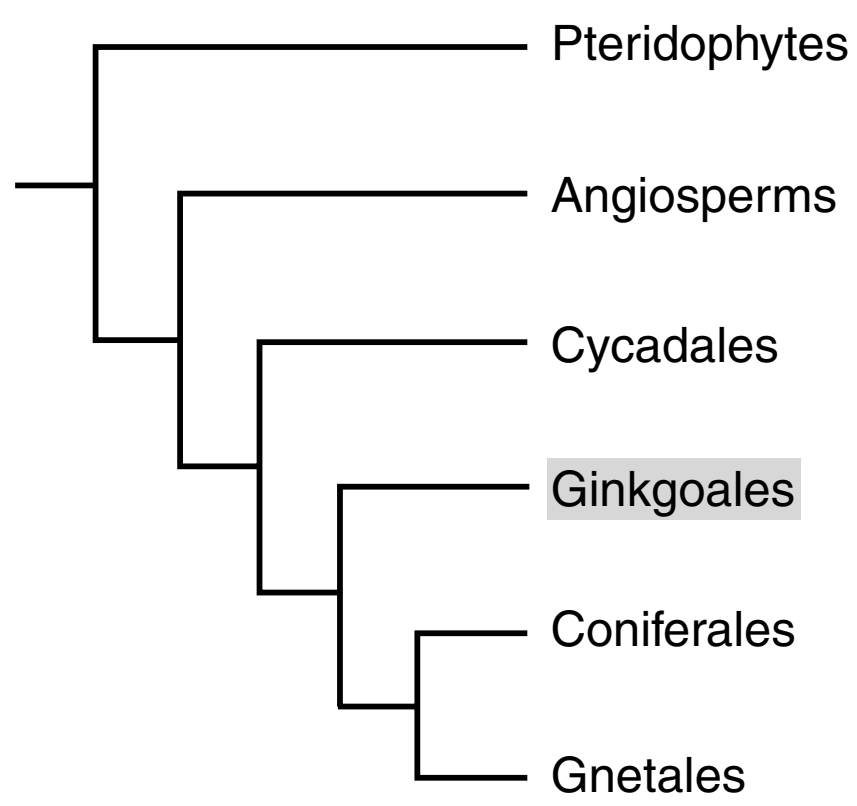

Figure I

Gene tree of extant gymnosperms. Ginkgo displays characters suggesting it forms a basal subgroup among the gymnosperms with cycads. Alternately Ginkgo is a sister group of the conifers. Here the placement of Ginkgo is shown as ambivalent between these two scenarios. 

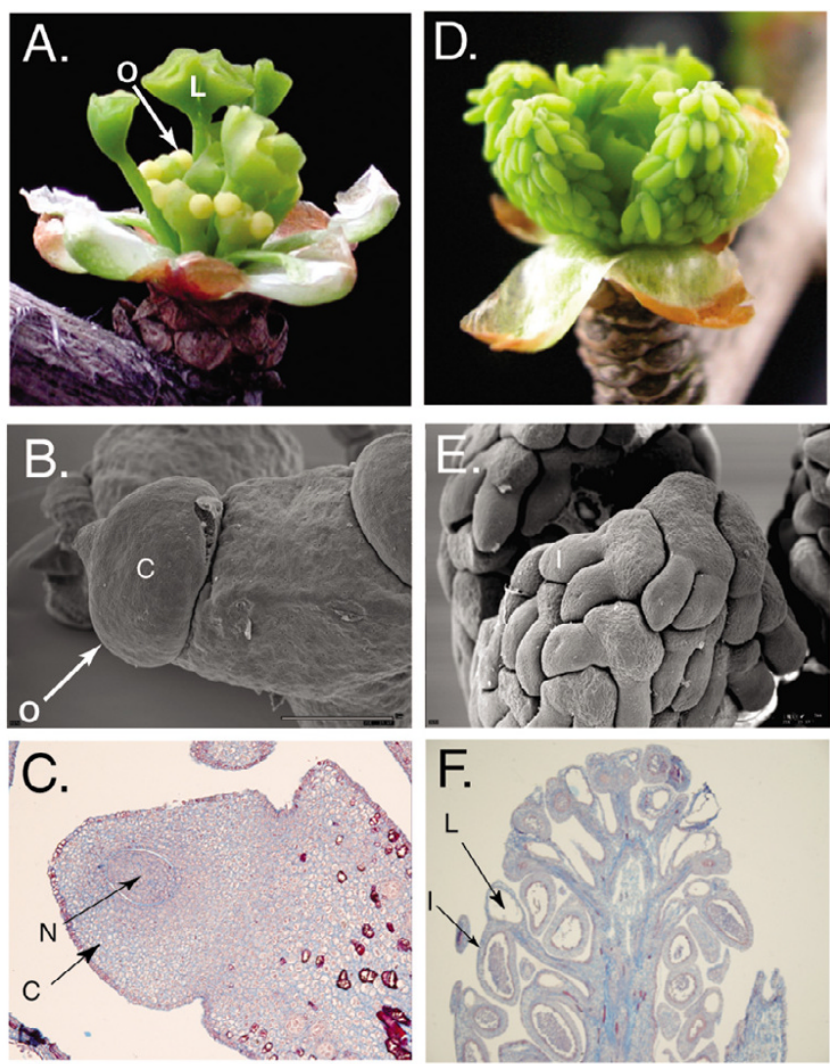

Figure 2

Ginkgo male and female short shoots. (A) The fertile female structure (megasporangia) has just emerged from the bud. Two ovules set on a green stalk are visible. Young, unfurled leaves, which have also emerged have extended above the megasporangia. The bracts, which originally enclosed the bud, are now completely opened below the leaves and megasporangia (B) Scanning EM of an ovule, which is completely enclosed by an integument. (C) A longitudinal cross section of the megasporangia reveals the integument enclosing the nucellus. (D) The male reproductive structure is a cluster of microsporangia. In the center of the bud are partly emerged leaves (E) Scanning EM shows two microsporangial lobes containing ripening pollen sacks attached to a stalk. $(\mathrm{F})$ Longitudinal cross section shows a large mucilage containing cavity juxtaposed from the microsporangia filled with immature pollen. $\mathrm{C}$ integument; $\mathrm{N}$, nucellus; I, microsporngia; L, mucilage cavity. $\mathrm{O}$, ovule

set of Ginkgo ESTs that were only common to our cycad EST dataset, further strengthening their classification as gymnosperm specific.

\section{Results and discussion Construction of a cDNA library from Ginkgo biloba fertile and vegetative tissue}

Young organs (Figure 2A and 2D) were collected during the spring from the opening buds of short shoots imme- diately after their emergence. At this stage, the megasporangium consists of an axis typically bearing two ovules (Figure 2A-C). The ovule is composed of a single integument surrounding a developing nucellus (Figure $2 \mathrm{C}$ ). The male structure consists of a main axis bearing two or more microsporangia (Figure 2D-F). RNA was extracted from the following organs: megagasporangia, microsporangia and two sets of leaves collected from either male or female trees. mRNA isolated from all four tissues was used to construct four separate cDNA libraries. (Both male and female leaf sequences were pooled during subsequent bioinformatic analysis). Size fractionation was used to enrich for full-length cDNAs during library construction. From this cDNA library, 6,434 sequence reads (Expressed Sequence Tags, ESTs) were generated. All Ginkgo biloba EST reads have been deposited in GenBank. It was determined that $3,739(58 \%)$ of the cDNA clones were over 500 bp long. 3618 of the reads were generated from the $5^{\prime}$ end of the CDNA, and 2816 were sequenced from the $3^{\prime}$ end. Cluster analysis on the EST sequence produced a unigene set of 3,830 contigs consisting of 2,851 singletons and 979 assemblies. Of the clusteredESTs, the longest contig was 2,172 bp. The entire unigene set or complete Ginkgo BLAST files can be downloaded at the following website [23]. Each G. biloba contig is given a numeric identifier. The constituent ESTs for each contig can be obtained at this website. Additional bioinformatic analysis of the Ginkgo biloba dataset can be accessed at the open Sputnik Comparative Genomics Platform at [24]. This site features sequence annotations, peptide sequence predictions, protein domain architectures and putative molecular markers (ISSRs) for the ginkgo EST derived unigenes. The sequence can be downloaded either as a fasta file, a clustered fasta file or as the derived peptide fasta file. In addition, BLAST analysis can be performed with the clustered ESTs from a given ginkgo organ against all genes in Arabidopsis thaliana or distinct plant clustered EST datasets using the ViCoGenTa program available at the New York Plant Genomics Consortium website [23].

\section{Ginkgo contig matches to genes in angiosperms, gymnosperms and non-seed plants}

TBLASTX (expect < 1eX10-5) was used to compare the G. biloba unigenes against all available plant ESTs from TIGR (The Institute for Genomic Research) [25], and the Plant Genome DataBase (Plant GDB) [26]. ESTs from these databases were downloaded and clustered into unigenes, which were used in the comparison. Next the Ginkgo unigene set was compared against the Arabidopsis and rice genome annotated protein sequences downloaded from TIGR. All genes used in this comparison against Ginkgo were divided into one of three taxonomically relevant categories: 1 . angiosperms, 2 . gymnosperms, and 3. non-seed plants. The angiosperm category encompasses all annotated rice and Arabidopsis genes 
A

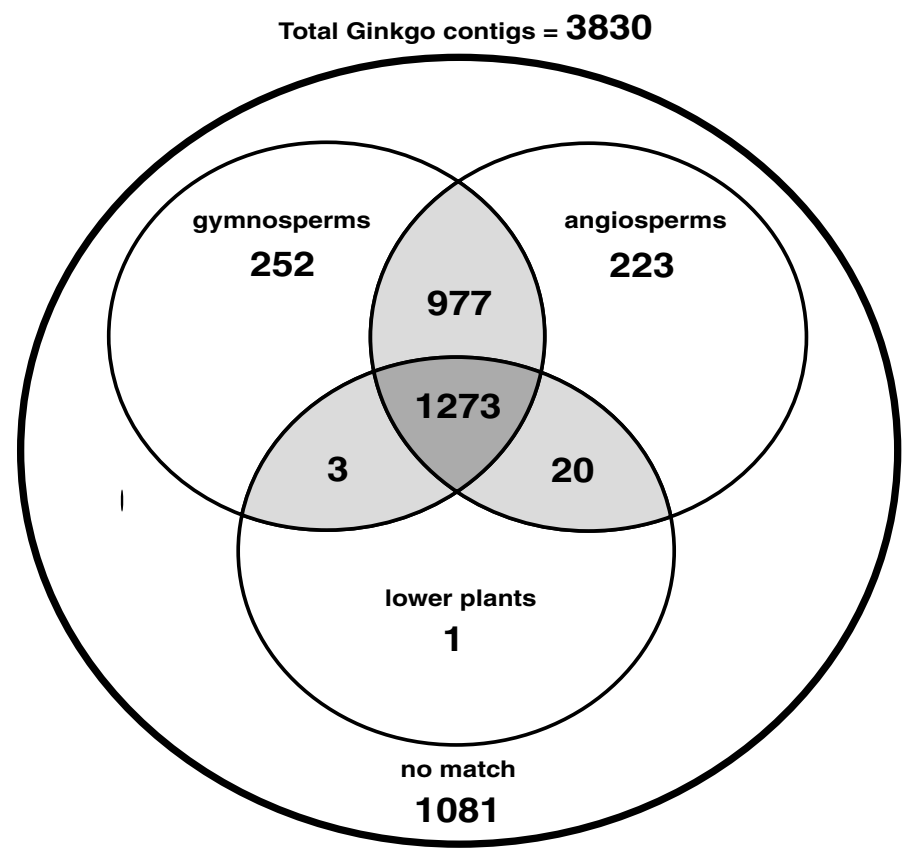

B

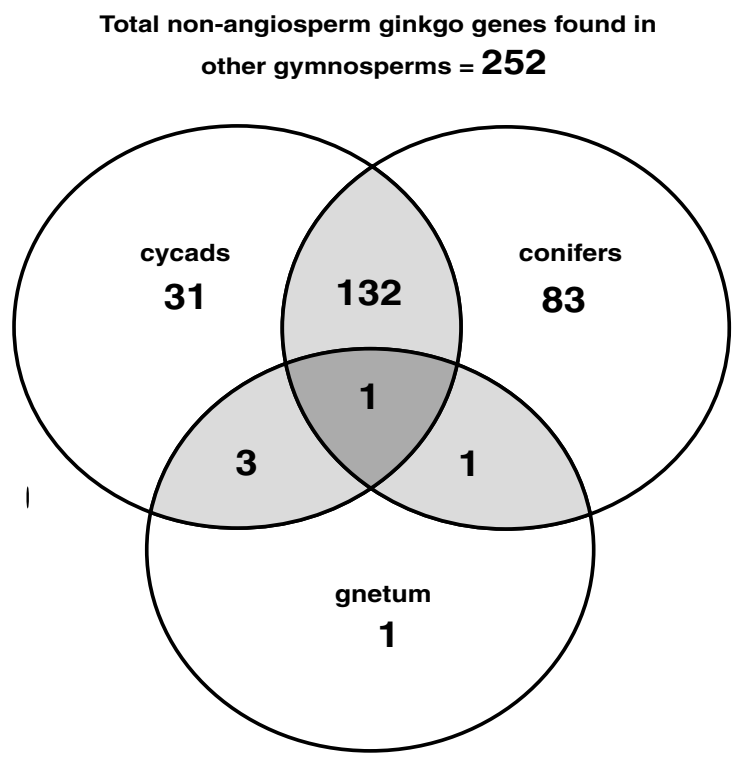

Figure 3

A Venn diagram illustrating the number of Ginkgo contigs with shared homology to genes found in non-seed plants, gymnosperms and/or angiosperms (A). A BLASTX E value >e 10-5 was used as a cut-off. (B) The Ginkgo contigs with similarity to gymnosperms but (no match to angiosperm genes) were further subgrouped according to their BLAST score homology (E value $>$ e 10-5) within gymnosperm taxa.

identified from their respective genomic sequences, as well as all higher plant ESTs. The majority of the gymnosperm ESTs came from the conifer groups pine and spruce but also include ESTs generated from the Plant Genomics Consortium containing ESTs from the two other gymnosperm clades, Cycadales and Gnetales. The non-seed plant category consisted of genes from all remaining plant ESTs including ferns, fern allies, bryophytes and algae available with the majority of the sequences originating from Physcomitrella patens and Chlamydamonas reinhardtii.

A Venn diagram shown in Figure 3A displays the number of Ginkgo contigs, which are shared between one or more of the plant EST datasets at low BLAST stringency value (expect < 1eX10-5). From the Venn diagram, it can be seen that a majority of Ginkgo unigenes (2749/3830) match genes in other plants, and 1081 have no match to other plant genes. Of those 2749 Ginkgo biloba unigenes with matches to other plant genes, a subgroup of 256 unigenes had no corresponding match to genes in the angiosperm dataset. Of these 256 Ginkgo genes that do not match angiosperms, 4 also match genes in non-seed plants.

The 252 Ginkgo biloba unigenes that only match gymnosperm genes were next partitioned into matches between the three other gymnosperm orders: Cycadales, Coniferales, and Gnetales (Figure 3B). Since there are significantly more conifer unigenes $(>60,000)$ than cycad unigenes (5459) that were used in this comparison, one would expect that the number of matches between Ginkgo and conifers would be significantly greater than matches between Ginkgo and cycads. However the actual number of matches between Ginkgo and conifers (215) is only slightly more then for Ginkgo and cycads (163). In other words despite the fact that there is over 10 times the number of conifer genes then cycad genes used in this comparison, Ginkgo matches to conifers are only 1.3 times greater then matches between Ginkgo and cycads. Of the matches between Ginkgo and gymnosperms, 31 match only cycads, (22 match Cycas rumphii, 6 match only uni- 
genes from the cycad species, Zamia furfuracea, (753 contigs deposited in Genbank from Brenner et al, unpublished data); and 3 Ginkgo contigs match unigenes from both Cycas rumphii and Zamia furfuracea).

As one might expect, unigenes with matches to other plants are somewhat longer then those that have no match to other plants. Of all Ginkgo unigenes with matches to other plants, $89 \%$ are greater than $300 \mathrm{bp}$, whereas $72 \%$ are greater than 300 bp then those Ginkgo unigenes with no matches to other plants.

\section{Common genes between cycads and Ginkgo}

Our comparative analysis of the Ginkgo EST dataset builds upon our results from a previous genomic study on the cycad, Cycas rumphii [18]. In our current analysis, three (CB090673:GinkgoA3816, CycadCB089620:GingkoA3730, and CycadCB089926:GinkgoA1532) of the fourteen unigenes from cycads previously found only among gymnosperms (after the full-length clone was sequenced), were also homologues to Ginkgo genes found only in gymnosperms. Considering the relatively small number of unigenes from Ginkgo $(3,830)$ and cycads $(4706)$ available for our comparative studies, the detection of the same gene match in Ginkgo and cycads with homology to only gymnosperms strengthens the argument that these genes are gymnosperms specific.

Ginkgo matches to non-seed plants but not angisosperms An additional four Ginkgo unigenes that are not found in angiosperms were detected in non-seed plants. Three of these Ginkgo unigenes (GinkgoA2411, GinkgoA3214 and GinkgoA325) match non-seed plants and other gymnosperm genes whereas the forth Ginkgo gene (GinkgoA2273) only matches non-seed plants with similarity to a gene in Chlamydomonas.

\section{Classification of G. biloba ESTs by functional categories}

Each contig from the Ginkgo dataset was automatically assigned to a functional category (FunCat) based on its top match against the MIPS FunCat list of functionally annotated gene sequences from $\mathrm{S}$. cerevisiae and $\mathrm{A}$. thaliana databases using BLASTP. A non-stringent expect value (E-value) of $<\mathrm{e}-10$ was chosen as the threshold. Table 1 below illustrates the relative fraction that each functional category comprises within the entire unigene set compared to our previous study in Cycas rumphii [18]. The four largest categories of Ginkgo ESTs according to this functional categorization are: "cellular organization" (19\%), "metabolism" (11\%), "unclassified proteins" $(14 \%)$, and "protein synthesis" (8\%). In general, these same categories are also the highest in the cycad EST library from our previous work, except for "protein synthesis", which appears increased in Ginkgo, whereas inter- estingly, the category of cell growth, cell division and DNA synthesis is reduced in Ginkgo compared to cycads.

\section{Ginkgo genes involved in development}

Analysis of the Ginkgo biloba dataset revealed a number of ESTs with highest BLAST similarity to genes with known roles in higher plant developmental processes. A sampling of some of these genes is shown below (Table 2 ). These genes included the Polycomb gene CURLY LEAF $[27,28]$ as well as LATERAL ORGAN BOUNDARIES (LOB) [29], EARLY FLOWERING 5 (ELF5) [30], FLOWERING LOCUS T (FT) [31], and CONSTANS (for review see [32] as well as five ESTs that match MADS box genes, some of which appear identical to previously cloned fragments of MADS genes from Ginkgo biloba including the G. biloba ortholog of AGAMOUS [19]. Other genes in our EST library have homologies to proteins that regulate development through protein turnover including SPA-1 [33]COP1 [34] and COP9 [35,36]. This sampling reveals that the EST dataset of Ginkgo biloba is a rich source of genes encoding proteins with known roles in development at the transcriptional as well as post-transcriptional stage.

\section{Conclusion}

The importance of Ginkgo for the study of plant evolution As the sole remaining species of an ancient genus of plants which has survived nearly 170 million years from the Jurassic [5], Ginkgo biloba is a taxonomic and geographic relict that may be even older because fossils displaying a "ginkgophyte" vegetative morphology have been found as early as the Permian [4]. Ginkgo has a number of plesiomorphic (unspecialized) as well as apomorphic (derived) traits that make it a valuable tool to study the evolution of seed plants. Here we used a genomic approach to investigate the genes involved in regulating development in Ginkgo by creating an EST library from both reproductive and vegetative tissues.

Similar to our previous analysis in Cycas rumphii, our Ginkgo EST study has found significant BLAST homology between Ginkgo ESTs with plant genes in gymnosperms and non-seed plants but not in angiosperms. Since ESTs, even when clustered in contiguous genes, may not represent the complete gene [37], often one will find homology to angiosperm genes when the remaining Ginkgo gene sequence is revealed. For example in the gymnosperm, Pinus taeda EST collection, contigs of increasing length have a higher likelihood then shorter contigs matching a known gene in the Arabidopsis genome [38]. However, in this same study a significant subcategory of very long contigs (>1900 bp) have no homology to Arabidopsis [38]. It is likely that at least some of these long contigs with no match to angiosperm genes represent full length genes that are specific to the gymnosperm and/or seed-less plant 
Table I: Placement of Ginkgo unigenes into functional categories (funcats). Ginkgo genes with a BLASTP expect value (E-value) of $>$ e- 10 were assigned into funcats based on their similarity score. The analysis was performed at the Centre for Biotechnology, Turku, Finland. Previous funcat analysis data from Cycas rumphii is shown.

\begin{tabular}{lcc}
\hline Functional Category & $\begin{array}{c}\text { Ginkgo } \\
\%\end{array}$ & Cycad\% \\
\hline Metabolism & 11.0 & 10.3 \\
Energy & 5.0 & 5.1 \\
Cell Growth, Cell Division and DNA Synthesis & 5.4 & 9.2 \\
Transcription & 3.6 & 5.0 \\
Protein Synthesis & 8.4 & 5.8 \\
Protein Destination & 5.8 & 8.3 \\
Transport Facilitation & 2.0 & 1.3 \\
Intracellular Transport & 3.4 & 4.3 \\
Cellular Biogenesis & 3.5 & 3.3 \\
Cellular Communication/Signal Transduction & 3.5 & 4.2 \\
Cell Rescue, Defense, Cell Death and Ageing & 7.2 & 6.8 \\
lonic Homeostasis & 0.5 & 0.1 \\
Cellular Organization & 18.8 & 21.6 \\
Classification Not Yet Clear-Cut & 7.3 & 4.6 \\
Unclassified Proteins & 14.1 & 10.3
\end{tabular}

clades. Our strategy to address this question involves screening for these same genes in additional taxa of gymnosperms, in the case of this study, Ginkgo biloba. In our analysis three Ginkgo genes that were only found in gymnosperms also matched the fourteen ESTs from our previous study of gymnosperm common cycad genes.

Along these same lines, our results suggest the presence of genes common to non-seed plants and gymnosperms that are not present in angiosperms. This non-seed plant/gymnosperm grouping is not surprising considering the fact that gymnosperms have morphologically common characters that are not found in the angiosperms - particularly in their reproductive structures. For example, the megagametophyte is highly reduced both in cell number and in structural organization in angiosperms when compared to gymnosperms. Although these results cannot say for certain that these genes are specific to non-seed plants and gymnosperms, or more specifically that these genes are found in gymnosperm structures that are not found in seed plant, it nonetheless represents an important starting point to correlate the presence or absence of gymnosperm genes in angiosperms and/or lower plants.

\section{Are cycads and Ginkgo sister taxa?}

One result from our study found that the number of Ginkgo contig matches to conifers are only 1.3 times greater then matches between Ginkgo and cycads despite the fact that there is over 10 times the number of conifer genes then cycad genes used in this comparison,. Taken together these results might indicate a closer evolutionary association between Ginkgo and cycads then between Ginkgo and conifers. This bias towards cycad/Ginkgo similarity correlates with the fact that the majority of molecular phylogenetic studies place as the cycads sister group to Ginkgo. Hopefully, this preliminary data will encourage further phylogenomic studies to fully resolve the hierarchy among extant gymnosperm orders. Until the full genome sequence becomes available for key gymnosperm taxa, EST sampling provides an important initial step for large scale identification of molecular markers to generate robust phylogenetic trees.

\section{Developmental regulators in Ginkgo}

In Ginkgo biloba we note here a variety of genes with similarity to developmental regulators in angiosperms. We also note below that homologues to some of these developmental regulators are also present in our Cycas rumphii library as either orthologs to those found in higher plants or at least, belonging to the same gene family. An EST from Ginkgo biloba that was detected in the megagametophyte library has high similarity to the Arabidopsis CURLY LEAF (CLC) gene, which belongs to the Polycomb-group proteins (PcGs). PcGs epigenetically regulate downstream target genes [28]. PcGs modify chromatinprotein complexes that repress homeotic gene transcription and influence cell proliferation. In Arabidopsis PcG genes have been shown to regulate MADS box genes [39]. The CLC protein product regulates the expression of AGAMOUS [27], a gene controlling floral organ identity [40]. Interestingly, an ortholog for angiosperm AGAMOUS was also detected in the Ginkgo megagametophyte library (Table 2). Ginkgo AGAMOUS, (previously named GBM5) was identified in a study where the MADS domains were examined in Ginkgo [19]. In this work Ginkgo AGAMOUS was shown via RT-PCR to be expressed in not only female but also in male and vegetative tissue. In our analysis, five total MADS box homologues were also detected in the Ginkgo EST dataset. Three of the Ginkgo ESTs from our library, GinkgoA2340, GinkgoA2730 and GinkgoA2850, are perfectly identical to the MADS domain gene fragments previously cloned by [19] as degenerate PCR products. The other two unigenes from our dataset have homologies to MADS genes (GinkgoA629 and GinkgoA352), but do not specifically match any of the PCR fragments isolated in their study. These two MADS box unigenes either do not include the small region amplified in their degenerate PCR screen or could alternative be unique MADS genes not isolated in their study. Unlike the degenerate primer approach used to isolate MADS genes, our EST approach offers the additional advantage of cloning entire genes or at least substantially large gene fragments. Among the few developmental genes examined in gymnosperms, most 
Table 2: Similarity match of Ginkgo unigenes to genes involved in development. The G. biloba unigene set was compared to Genbank using a BLASTP cut-off score < e-5. The top match is listed under subject description. The organ(s) from which the listed ESTs were detected are: $\mathbf{G}=$ megagametophyte, $I=$ microgametophyte, and $L=$ leaf.

\begin{tabular}{|c|c|c|c|}
\hline \multicolumn{4}{|c|}{ Genes in Ginkgo biloba with similarity to developmental genes in other plants } \\
\hline Contig id. & organ & BLAST homology match & E-value \\
\hline A2725 & G & gi| $|903019|$ emb|CAA7|599.1| curly leaf [A. thaliana] & $4.00 \mathrm{E}-37$ \\
\hline A3095 & I & gi| I523 |388|ref|NP_I8800I.I| LOB domain family protein [A. thaliana] & $2.00 \mathrm{E}-21$ \\
\hline A28I5 & I & gi|4254II56|gb|AASI947I.I| EARLY FLOWERING 5 [A. thaliana] & I.00E-33 \\
\hline A335I & 1 & gi|4903 I39|dbj|BAA77836.I| extensive homology to FT (FLOWERING LOCUS T, AB027504) & $4.00 \mathrm{E}-10$ \\
\hline A24I & $\mathrm{I}, \mathrm{L}$ & gi|4l323976|gb|AAS00054.1| CONSTANS-like protein COI [Populus deltoides] & $1.00 \mathrm{E}-30$ \\
\hline Al59I & $\mathrm{L}$ & gb|AAG43405.I|AFI7293I_I homeobox I [Picea abies] & $5.00 \mathrm{E}-23$ \\
\hline Al591 & G & gi| $|47| 5|83|$ emb|CAC44080.1| putative MADS-domain transcription factor DEFH7 [A. majus] & $2.00 \mathrm{E}-39$ \\
\hline A2737 & G & gi|30230270|gb|AAM76208.I| AGAMOUS-like MADS-box transcription factor [Ginkgo biloba] & $5.00 \mathrm{E}-28$ \\
\hline $\mathrm{A} 2850$ & 1 & gi|253079 |8|pir||S5 I 935 probable MADS-box protein dall - Norway spruce dall [Picea abies] & 5.00E-72 \\
\hline A629 & G, L & gi|7446554|pir||TI075I MADS-box protein MADS9 - Monterey pine & $6.00 \mathrm{E}-17$ \\
\hline A352 & $\mathrm{G}, \mathrm{I}$ & gi|7446559|pir||T0957। MADS box protein MADS2 - Monterey pine & $4.00 \mathrm{E}-75$ \\
\hline A914 & $\mathrm{G}, \mathrm{I}$ & gi| I840 I 293|ref|NP_565632.I| COP9 / CSN signalosome complex subunit [A. thaliana] & $1.00 \mathrm{E}-45$ \\
\hline A2730 & G & gi|I5225760|ref|NP_I80854.I| COPI regulatory protein [A. thaliana] & $2.00 \mathrm{E}-60$ \\
\hline A944 & G, I & gi|30694320|ref|NP_849784.I|argonaute protein(AG0I) [A. thaliana] & I.00E-56 \\
\hline
\end{tabular}

attention has focused on the expression of MADS homologs $[41,42]$.

Other developmental genes found in the Ginkgo EST library include those with homology to regulators of flowering such as EARLY FLOWERING 5 (ELF5), which controls the levels of the gene FLC, which itself is a central regulator of flowering [30]. Another Ginkgo EST includes FLOWERING LOCUS T (FT), which belongs to a small family of genes (FT/TFL1) that act to promote flowering as a downstream component from CONSTANS [43]. CONSTANS is a transcription factor that has a critical role integrating circadian rhythms and light signals (for review see [32]). As one would expect an EST homolog for the CONSTANS gene family was found in Ginkgo. CONSTANS belongs to a large gene family, which may have redundant roles in plants [44]. Not surprisingly, we also found homologs to CONSTANS in our previous study on cycad leaf ESTs [18]. In that flowering plants are believed to have evolved from gymnosperms, a survey of CONSTANS, ELF, and FT in gymnosperms, particularly in very young reproductive tissue might help define the origins of reproductive induction in non-flowering plants. Among the other genes related to developmental regulators includes a homologue to LATEROL ORGAN BOUNDARIES (LOB) domain gene family which in Arabidopsis has over 40 members [29]. The molecular mechanism of $L O B$ domain containing genes is unknown, but one gene in Arabidopsis, ASYMMETRIC LEAVES2, is required for normal leaf development, by potentially acting as a regulatory repressor of KNOX genes [45]. A KNOX homolog is also present in our EST library and was found in male reproductive tissues and $H O X$ genes were also detected in our previous analysis in C. rumphii

Another important component regulating development occurs at the level of protein degradation. A gene recognized in our EST library includes COP1. COP1, serves as an E3 ubiquitin targeting photomorphogenic factors such as HY5 for degradation [33]. Another Ginkgo EST from the library has highest similarity to COP9. In our previous EST analysis in Cycas rumphii an EST was also isolated with similarity to COP9 [18]. COP9 is a subunit of the COP9 signalosome complex that controls multiple signaling pathways that regulate development in all eukaryotes $[35,36]$. In Arabidopsis, the cop9 and cop1 mutants are constitutively photomorphogenic in dark grown seedlings [46]. Unlike angiosperms, seedlings from conifers are constitutively photomorphogenic when grown in the dark $[47,48]$. In Ginkgo, chlorophyll and chloroplast development is completely dependent on light, however this process proceeds at a markedly slower pace then in flowering plants. That is, photomorphogenic development in Ginkgo seedlings is strongly delayed after transfer from dark grown conditions to light grown conditions when compared to seed plants $[20,21]$. The dark grown phenotype of cycads is unreported. Considering this variability in photomorphic development among and between the gymnosperms and the angiosperms, the discovery of genes encoding photomorphogenic regulators in gymnosperms will help understand the evolution of photomorphogenesis in seed plants. 
Taken together, our genomics analysis of Ginkgo biloba is an important additional step to analyze the role of molecular development of early seed plants. Thus the stage is set to further determine the role of these genes during the development of ancillary structures found between Ginkgo, cycads and other gymnosperms with higher plants as well as the role of those in structures that are unique to gymnosperms and/or the non-seed plants as a step to understand the evolution of the seed plant habit.

\section{Methods}

\section{Tissue collection and library construction and DNA purification}

Newly emerged microsporangia from accession 76163B, megasporangia from accession $76163 \mathrm{D}$, and immature leaves from both accessions were collected from newly opened buds of Ginkgo biloba growing in the New York Botanical Garden outdoor collection on April 12, 2002. Organs were snap frozen in liquid nitrogen. RNA was collected from each organ and a cDNA library was constructed from fractionated cDNA according to [49].

\section{Microscopy}

Ginkgo apices were collected on April 19. Bract tissues were removed from the apex leaving the leaves and reproductive tissue, which was fixed in FAA (50\% ethanol, 5\% glacial acetic Acid, 3.7\% formaldehyde) under vacuum (20 In. Hg) at room temperature. Fresh FAA was vacuum infiltrated two additional times. Tissue was stored in $70 \%$ ethanol at $4^{\circ} \mathrm{C}$.

For histology, tissue was prepared by sequential (overnight $4 \mathrm{C}$ incubation at each alcohol grade) dehydration in 80, 90, 95 and finally 100\% ethanol plus Eosin Y (National Medicinal Products) followed by two treatments in 100\% ethanol for 2 hours at room temperature. The tissue was next placed in a 1:1 solution of ethanol and toluene, then twice in toluene alone, each time for 2 hours at room temperature. The tissue was then placed in toluene with a quarter-volume of paraffin (PARAPLAST X$\mathrm{TRA}^{\circledast}$ (Fisher)) chips at $60^{\circ} \mathrm{C}$ overnight. The tissue was then embedded in melted paraffin with six wax changes over the course of three days at $55^{\circ} \mathrm{C}$. Apices were sectioned on a MICROM HM 355 microtome. $8 \mu \mathrm{m}$ thick sections were taken using a blade angle of $9^{\circ}$. The tissue was stained with Astra Blue and Safranin. After mounting on slides, sections were imaged using a Nikon DXM1200F digital microscope camera.

For scanning electron microscopy, fixed materials were dissected, dehydrated in ethanol and critical point dried in a Denton critical point dryer. Dried materials were affixed to aluminium E. M. stubs and coated with between 80-240 A of palladium in a Hummer II Sputter Coater. Coated materials were then observed using a Jeol scan- ning electron microscope at 15 or $20 \mathrm{kV}$. Images were digitally recorded and evaluated using Adobe Photoshop 9.0.

\section{EST sequencing and gene analysis}

Plasmid DNA was collected as described in the manual (Stratagene), catalogue number 200450 in the in vivo mass excision section. Sequence analysis was performed at CSHL using an ABI 3700 Capillary sequencer for separation and nucleotide detection. Reactions were performed using a 1/16 Big Dye Terminator. Sequencing was performed with either the -21 M13 forward and/or reverse primer. ESTs were assembled using Phrap [50,51] and clustered into contigs using the CAP3 program [52]

\section{Peptide extraction}

Peptide sequences were derived for all unigenes using the ESTScan application [53] run with the default parameters. Prior to the ESTScan predictions, a Ginkgo species-specific ESTScan model was created. ESTScan was trained with Ginkgo ORFs identified from the best match of BLASTX analyses performed on the unigene sequence against the Swissprot protein database. All BLASTX matches were filtered using the arbitrary expectation value of $1 \mathrm{e}-10$.

\section{Sequence annotation}

Sequence annotation on each of the Ginkgo cluster consensus sequences and derived peptides were performed within the openSputnik application [54]. Results were assessed for possible contamination by searching for homology to the E. coli and human genomes and were scored for homology to a wide range of non-coding RNAs and plant chloroplast and mitochondrial genomes. Homology searches were performed using the BLAST application [55] and results were filtered using the expectation value $<1 \mathrm{e}-10$. Functional assignment was performed on both cluster consensus sequence and the peptide sequence. Assignments were made using BLASTX and BLASTP respectively against the MIPS catalogue of functionally assigned proteins (funcat) $[50,51]$, tentative functional assignments were filtered using the expectation value $<1 e-10$.

\section{Categorization of Ginkgo contigs}

All Ginkgo contigs sequences were aligned against a PlantEST database using TBLASTX [55] and BLASTX against the NR (aa) database. The PlantEST database was created by downloading all plant ESTs in GenBank and assembling them using Phrap [50,51]. Todd Wood from Clemson University provided the PERL script that creates the PlantEST databases as described above. The NR (aa) database is a non-redundant database of protein sequences from GenBank. 


\section{Determination of gymnosperm specific genes}

All available plant ESTs were downloaded from GenBank and separated into three datasets consisting of angiosperms (monocots and dicots), gymnosperms, or non-seed plants (ferns, mosses and algae). Downloaded ESTs were assembled using Phrap [50,51]. All matches with an expect value $<1$ e 10-5 are considered significant.

\section{Authors' contributions}

EB conceived of this project. He participated in its design, experiments and drafted the manuscript. DS and GC also conceived this project and participated in its design and coordination. MK played the major role in the bioinformatics analysis. SAR performed the funcat analysis and built the Sputnik website, AD performed the scanning electron microscopy work, WM and GS performed the histological sectioning, RT and SJR performed the cDNA library construction, RM facilitated the EST sequencing. All authors read and approved the final manuscript.

\section{Acknowledgements}

We would like to thank Vivekanand Balija for sequence generation. Funding for this work comes from the Plant Genomics Consortium. The plant Genomics Consortium is made possible by the generosity of the Altria Group, Inc., The Mary Flagler Cary Charitable Trust, The Eppley Foundation for Research, Inc., The Ambrose Monell Foundation, The Wallace Genetic Foundation, Inc., and the National Science Foundation Plant Genome Group grant number DBI-0421604.

\section{References}

I. Hori T, Ridge RW, Tulecke W, Del Tredici P, Tremouillaux-Guiller J, Tobe H: Ginkgo biloba-A Global Treasure. Tokyo, Springer; 1997.

2. Curtis-Prior P, Vere D, Fray P: Therapeutic value of Ginkgo biloba in reducing symptoms of decline in mental function. J Pharm Pharmacol 1999, 5 I:535-54I.

3. Gold PE, Cahill L, Wenk GL: The lowdown on Ginkgo biloba. Sci Am 2003, 288:86-9l.

4. Rothwell GW, Holt B: Fossils and phenology in the evolution of Ginkgo biloba. In Ginkgo-biloba-A Global Treasure Edited by: Hori T, Ridge RW, Tulecke W, Del Tredici P, Trémouillaux-Guiller J and Tobe H. Tokyo, Springer-Verlag; 1997:223-230.

5. Zhou Z, Zheng S: The missing link in Ginkgo evolution. Nature 2003, 423:821-822

6. Fagard M, Boutet S, Morel JB, Bellini C, Vaucheret H: AGOI, QDE2 , and RDE-I are related proteins required for post-transcriptional gene silencing in plants, quelling in fungi, and RNA interference in animals. Proc Natl Acad Sci USA 2000, 97: I1650-11654.

7. Doyle JA, Donoghue MJ: Seed plant phylogeny and the origin of angiosperms: an experimental cladistic approach. Botanical Reviews 1986, 52:321-43I.

8. Donoghue MJ, Doyle JA: Seed plant phylogeny: Demise of the anthophyte hypothesis? Curr Biol 2000, I0:R I06-9.

9. Magollón S, Sanderson MJ: Relationships among seed plants inferred from highly conserved genes: sorting confilicting phylogenetic signals among ancient lineages. Am J Bot 2002, 89:199|-2006.

10. Chaw SM, Parkinson CL, Cheng Y, Vincent TM, Palmer JD: Seed plant phylogeny inferred from all three plant genomes: monophyly of extant gymnosperms and origin of Gnetales from conifers. Proc Natl Acad Sci USA 2000, 97:4086-409l.

II. Bowe LM, Coat G, dePamphilis CW: Phylogeny of seed plants based on all three genomic compartments: extant gymnosperms are monophyletic and Gnetales' closest relatives are conifers. Proc Natl Acad Sci USA 2000, 97:4092-4097.
12. Hasebe M: Molecular phylogeny of Ginkgo biloba: close relationship between Ginkgo biloba and cycads. In Ginkgo biloba-a Global Treasure Edited by: Hori T, Ridge RW, Tulecke W, Del Tredici $\mathrm{P}$, Trémouillaux-Guiller J and Tobe H. Tokyo, Springer-Verlag; 1997:173-181.

13. Chamberlain C): Gymnosperms. Structure and Evolution. Chicago, University of Chicaco Press; 1935.

14. Friedman WE: Morphogenesis and experimental aspects of growth and development of the male gametophyte of Ginkgo biloba in vitro. American Journal of Botany 1987, 74:1816-1830.

15. Ikeno S, Hirase S: Spermatozoids in gymnosperms. Ann of Bot I897, I I :344-345.

16. Foster AS, Gifford EM: Comparative Morphology of Vascular Plants. second edition edition. Edited by: Kennedy D and Park RB. San Francisco, W. H. Freeman and Company; 1974.

17. Zhang P, Tan HT, Pwee KH, Kumar PP: Conservation of class C function of floral organ development during 300 million years of evolution from gymnosperms to angiosperms. Plant J 2004, 37:566-577.

18. Brenner ED, Stevenson DW, McCombie RW, Katari MS, Rudd SA, Mayer KF, Palenchar PM, Runko SJ, Twigg RW, Dai G, Martienssen RA, Benfey PN, Coruzzi GM: Expressed sequence tag analysis in Cycas, the most primitive living seed plant. Genome Biol 2003, 4:R78.

19. Jager M, Hassanin A, Manuel M, Guyader HL, Deutsch J: MADS-box genes in Ginkgo biloba and the evolution of the AGAMOUS Family. Mol Biol Evol 2003, 20:842-854.

20. Chinn E, Silverthorne J: Light-dependent chloroplast development and expression of a light-harvesting chlorophyll $a / b$ binding protein gene in the gymnosperm Ginkgo biloba. Plant Physiol 1993, 103:727-732.

21. Chinn E, Silverthorne J, Hohtola A: Light-regulated and organspecific expression of types I, 2, and 3 light-harvesting complex b mRNAs in Ginkgo biloba. Plant Physiol 1995, 1 07:593-602.

22. Bierhorst DW: Morphology of Vascular Plants. , Macmillan; I97I.

23. The New York Plant Genomics Consortium [http://nypge nomics.org]

24. OpenSputnik Comparative Genomics Platform [http://sput nik.btk.fi/]

25. The Institute for Genomic Research [http://www.tigr.org/]

26. Plant Genomic Database [http://www.plantgdb.org/]

27. Goodrich J, Puangsomlee P, Martin M, Long D, Meyerowitz EM, Coupland G: A Polycomb-group gene regulates homeotic gene expression in Arabidopsis. Nature 1997, 386:44-5I.

28. Kohler C, Grossniklaus U: Epigenetic inheritance of expression states in plant development: the role of Polycomb group proteins. Curr Opin Cell Biol 2002, 14:773-779.

29. Shuai B, Reynaga-Pena CG, Springer PS: The lateral organ boundaries gene defines a novel, plant-specific gene family. Plant Physiol 2002, I 29:747-76I.

30. Noh YS, Bizzell CM, Noh B, Schomburg FM, Amasino RM: EARLY FLOWERING 5 acts as a floral repressor in Arabidopsis. Plant J 2004, 38:664-672.

31. Weigel D, Ahn JH, Blazquez MA, Borevitz JO, Christensen SK Fankhauser C, Ferrandiz C, Kardailsky I, Malancharuvil EJ, Neff MM, Nguyen JT, Sato S, Wang ZY, Xia Y, Dixon RA, Harrison MJ, Lamb CJ, Yanofsky MF, Chory J: Activation tagging in Arabidopsis. Plant Physiol 2000, I22:1003-1013.

32. Hayama R, Coupland $G$ : The molecular basis of diversity in the photoperiodic flowering responses of Arabidopsis and rice. Plant Physiol 2004, I 35:677-684.

33. Saijo Y, Sullivan JA, Wang H, Yang J, Shen Y, Rubio V, Ma L, Hoecker $U$, Deng XW: The COPI-SPAI interaction defines a critical step in phytochrome A-mediated regulation of HY 5 activity. Genes Dev 2003, I 7:2642-2647.

34. Seo HS, Yang JY, Ishikawa M, Bolle C, Ballesteros ML, Chua NH: LAF I ubiquitination by COPI controls photomorphogenesis and is stimulated by SPAI. Nature 2003, 423:995-999.

35. Chamovitz DA, Glickman M: The COP9 signalosome. Curr Biol 2002, I 2:R232.

36. Hellmann H, Estelle M: Plant development: regulation by protein degradation. Science 2002, 297:793-797.

37. Rudd S: Expressed sequence tags: alternative or complement to whole genome sequences? Trends Plant Sci 2003, 8:321-329. 
38. Kirst M, Johnson AF, Baucom C, Ulrich E, Hubbard K, Staggs R, Paule $C$, Retzel E, Whetten R, Sederoff R: Apparent homology of expressed genes from wood-forming tissues of loblolly pine (Pinus taeda L.) with Arabidopsis thaliana. Proc Natl Acad Sci U SA 2003, 100:7383-7388.

39. Katz A, Oliva M, Mosquna A, Hakim O, Ohad N: FIE and CURLY LEAF polycomb proteins interact in the regulation of homeobox gene expression during sporophyte development. Plant J 2004, 37:707-719.

40. Bowman JL, Smyth DR, Meyerowitz EM: Genetic interactions among floral homeotic genes of Arabidopsis. Development 1991, II 2:1-20.

41. Brenner ED, Stevenson DW: Genomic Approaches to Understand the Origins of Seeds. In Landscapes, Genomics and Transgenic Conifers Edited by: Williams CG. Amsterdam, Publisher: KluwerSpringer; 2005

42. Theissen G, Becker A, Di Rosa A, Kanno A, Kim JT, Munster T, Winter $\mathrm{KU}$, Saedler $\mathrm{H}$ : A short history of MADS-box genes in plants. Plant Mol Biol 2000, 42: I I5-I49.

43. Kardailsky I, Shukla VK, Ahn JH, Dagenais N, Christensen SK, Nguyen JT, Chory J, Harrison MJ, Weigel D: Activation tagging of the floral inducer FT. Science 1999, 286: 1962-1965.

44. Lagercrantz $U$, Axelsson $T$ : Rapid evolution of the family of CONSTANS LIKE genes in plants. Mol Biol Evol 2000, 17:1499-I507.

45. Lin WC, Shuai B, Springer PS: The Arabidopsis LATERAL ORGAN BOUNDARIES-domain gene ASYMMETRIC LEAVES2 functions in the repression of KNOX gene expression and in adaxial-abaxial patterning. Plant Cell 2003, I 5:224I-2252.

46. Suzuki G, Yanagawa Y, Kwok SF, Matsui M, Deng XW: Arabidopsis COPIO is a ubiquitin-conjugating enzyme variant that acts together with COPI and the COP9 signalosome in repressing photomorphogenesis. Genes Dev 2002, 16:554-559.

47. Bogdanovic M: Chlorophyll formation in the dark. Physiol Plant 1973, 29:17-18.

48. Peer W, Silverthorne J, Peters JL: Developmental and light-regulated expression of individual members of the light-harvesting complex b gene family in Pinus palustris. Plant Physiol 1996, I I I:627-634.

49. Brenner ED, Stevenson DW, Twigg RW: Cycads: evolutionary innovations and the role of plant-derived neurotoxins. Trends Plant Sci 2003, 8:446-452.

50. Ewing B, Green P: Base-calling of automated sequencer traces using phred. II. Error probabilities. Genome Res 1998 8:186-194.

5I. Ewing B, Hillier L, WendI MC, Green P: Base-calling of automated sequencer traces using phred. I. Accuracy assessment. Genome Res 1998, 8: 175-185.

52. Huang $X$, Madan A: CAP3: A DNA sequence assembly program. Genome Res 1999, 9:868-877.

53. Iseli C, Jongeneel CV, Bucher P: ESTScan: a program for detecting, evaluating, and reconstructing potential coding regions in EST sequences. Proc Int Conf Intell Syst Mol Biol 1999: 138-148.

54. Rudd S: openSputnik--a database to ESTablish comparative plant genomics using unsaturated sequence collections. Nucleic Acids Res 2005, 33 (Database Issue):D622-7.

55. Altschul SF, Gish W, Miller W, Myers EW, Lipman DJ: Basic local alignment search tool. J Mol Biol 1990, 21 5:403-4I0.
Publish with Biomed Central and every scientist can read your work free of charge

"BioMed Central will be the most significant development for disseminating the results of biomedical research in our lifetime. "

Sir Paul Nurse, Cancer Research UK

Your research papers will be:

- available free of charge to the entire biomedical community

- peer reviewed and published immediately upon acceptance

- cited in PubMed and archived on PubMed Central

- yours - you keep the copyright
BioMedcentral 